\title{
Differences of Composition Operators on Bloch Type Spaces
}

Qin Huang*

Fujian Commercial College, Fuzhou 350003, Fujian, P.R. China.

Received 8 August 2015; Accepted (in second revised version) 27 December 2015

\begin{abstract}
In 2007, T. Hosokawa and S. Ohno gave the sufficient and necessary conditions of the boundedness and compactness of differences of composition operators on the Bloch space. On this base, this paper will generalize these conditions of the boundedness and compactness of differences of composition operators on the Bloch type space.
\end{abstract}

AMS subject classifications: 52B10, 65D18, 68U05, 68U07

Key words: Bloch type spaces, differences of composition operators, boundedness, compactness.

\section{Introduction}

Let $\mathbb{D}=\{z:|z|<1\}$ be the open unit disk in the complex plane $\mathbf{C}$. Let $H(\mathbb{D})$ denote the set of all analytic functions in $\mathbb{D}$ and $S(\mathbb{D})$ the set of analytic self-maps of $\mathbb{D}$. For any self-map $\varphi \in S(\mathbb{D})$, it induces the composition operator $C_{\varphi}$ defined by

$$
C_{\varphi} f=f \circ \varphi, f \in H(\mathbb{D}) .
$$

For $0<\alpha<\infty$, a function $f \in H(\mathbb{D})$ is said to belong to the $\alpha$-Bloch space $\mathscr{B}^{\alpha}$ if

$$
\|f\|_{\alpha}=\sup _{z \in \mathbb{D}}\left(1-|z|^{2}\right)^{\alpha}\left|f^{\prime}(z)\right|<\infty
$$

and to the little $\alpha$-Bloch space $\mathscr{B}_{0}^{\alpha}$ if $f \in \mathscr{B}^{\alpha}$ and

$$
\lim _{|z| \rightarrow 1}\left(1-|z|^{2}\right)^{\alpha}\left|f^{\prime}(z)\right|=0
$$

For $z, w \in \mathbb{D}$, let $\alpha_{w}$ be the Möbius transformation of $\mathbb{D}$ defined by

$$
\alpha_{w}(z)=\frac{w-z}{1-\bar{w} z}
$$

${ }^{*}$ Corresponding author. Email address: tianlan1983@163.com (Q. Huang) 
and the pseudo-hyperbolic distance between $z$ and $w$ is given by

$$
\rho(z, w)=\left|\alpha_{w}(z)\right| .
$$

We also need the following induced distance

$$
d_{\alpha}(z, w)=\sup _{f \in \mathscr{B}^{\alpha},\|f\|_{\alpha} \leq 1}\left|\left(1-|z|^{2}\right)^{\alpha} f^{\prime}(z)-\left(1-|w|^{2}\right)^{\alpha} f^{\prime}(w)\right| .
$$

For $0<\alpha, \beta<\infty, \varphi \in S(\mathbb{D})$, we use the short-hand notation

$$
\mathscr{D}^{\alpha, \beta} \varphi(z)=\frac{\left(1-|z|^{2}\right)^{\beta}}{\left(1-|\varphi(z)|^{2}\right)^{\alpha}} \varphi^{\prime}(z), \quad z \in \mathbb{D} .
$$

When $\alpha=\beta=1, \mathscr{D}^{\alpha, \beta} \varphi$ becomes the Schwarz-Pick type derivative of $\varphi$.

The difference of composition operators on the Bloch space has been studied in [1] and [4]. In this paper, we study the problem on the $\alpha$-Bloch spaces. One important application of differences of composition operators is to study the topological structure of the space of composition operators, which will be considered in another paper.

\section{Boundedness of $C_{\varphi}-C_{\psi}$}

In this section we give necessary and sufficient conditions for the differences of composition operators from $\mathscr{B}^{\alpha}$ to $\mathscr{B}^{\beta}$ for $0<\alpha, \beta<\infty$.

Lemma 2.1. ([8]) For $z, w \in \mathbb{D}, 0<\alpha<\infty$, there exists a constant $c$ independent of $z, w$ such that

$$
d_{\alpha}(z, w) \leq c \rho(z, w) .
$$

Theorem 2.1. For $0<\alpha, \beta<\infty$ and $\varphi, \psi \in S(\mathbb{D})$, the following statements are equivalent:

(1) $C_{\varphi}-C_{\psi}: \mathscr{B}^{\alpha} \rightarrow \mathscr{B}^{\beta}$ is bounded;

(2) $C_{\varphi}-C_{\psi}: \mathscr{B}_{0}^{\alpha} \rightarrow \mathscr{B}^{\beta}$ is bounded;

(3)

$$
\sup _{z \in \mathbb{D}}\left|\mathscr{D}^{\alpha, \beta} \varphi(z)-\mathscr{D}^{\alpha, \beta} \psi(z)\right|<\infty
$$

and

$$
\sup _{z \in \mathbb{D}}\left|\mathscr{D}^{\alpha, \beta} \varphi(z)\right| \rho(\varphi(z), \psi(z))<\infty
$$

(4)

$$
\sup _{z \in \mathbb{D}}\left|\mathscr{D}^{\alpha, \beta} \varphi(z)-\mathscr{D}^{\alpha, \beta} \psi(z)\right|<\infty
$$

and

$$
\sup _{z \in \mathbb{D}}\left|\mathscr{D}^{\alpha, \beta} \psi(z)\right| \rho(\varphi(z), \psi(z))<\infty .
$$


Proof. When either $\varphi$ or $\psi$ is constant, by [2] we have the assertion, so we may assume that both $\varphi$ and $\psi$ are nonconstant.

(1) implies (2) is obvious.

(2) implies (3). For $\lambda \in \mathbb{D}$ with $\lambda \neq 0$, let

$$
\begin{aligned}
& f_{\lambda}(z)=\frac{1}{2^{\alpha+1}} \frac{1-|\lambda|^{2}}{\alpha \bar{\lambda}(1-\bar{\lambda} z)^{\alpha}} \\
& g_{\lambda}(z)=\frac{1-|\lambda|^{2}}{(\alpha+1) 2^{\alpha+1}}\left(\frac{\lambda-z}{\bar{\lambda}(1-\bar{\lambda} z)^{\alpha+1}}+\frac{1}{\alpha \bar{\lambda}^{2}(1-\bar{\lambda} z)^{\alpha}}\right) .
\end{aligned}
$$

Then $f_{\lambda}, g_{\lambda} \in \mathscr{B}_{0}^{\alpha}$ and

$$
\begin{aligned}
& \left\|f_{\lambda}\right\|_{\alpha}=\sup _{z \in \mathbb{D}} \frac{\left(1-|z|^{2}\right)^{\alpha}}{2^{\alpha+1}} \frac{1-|\lambda|^{2}}{|1-\bar{\lambda} z|^{\alpha+1}} \leq 1 \\
& \left\|g_{\lambda}\right\|_{\alpha}=\sup _{z \in \mathbb{D}} \frac{\left(1-|\lambda|^{2}\right)\left(1-|z|^{2}\right)^{\alpha}}{(\alpha+1) 2^{\alpha+1}}\left|\frac{(\alpha+1)(\lambda-z)}{(1-\bar{\lambda} z)^{\alpha+2}}\right| \leq 1 .
\end{aligned}
$$

For $w \in \mathbb{D}$ with $\varphi(w) \neq 0$, we have

$$
\begin{aligned}
\infty & >\left\|C_{\varphi}-C_{\psi}\right\| \geq\left\|\left(C_{\varphi}-C_{\psi}\right) f_{\varphi(w)}\right\|_{\beta}=\sup _{z \in \mathbb{D}}\left(1-|z|^{2}\right)^{\beta}\left|\left(\left(C_{\varphi}-C_{\psi}\right) f_{\varphi(w)}\right)^{\prime}(z)\right| \\
& \geq\left(1-|w|^{2}\right)^{\beta}\left|\left(\left(C_{\varphi}-C_{\psi}\right) f_{\varphi(w)}\right)^{\prime}(w)\right| \\
& =\frac{1}{2^{\alpha+1}}\left|\left(1-|w|^{2}\right)^{\beta} \frac{1-|\varphi(w)|^{2}}{\left(1-|\varphi(w)|^{2}\right)^{\alpha+1}} \varphi^{\prime}(w)-\left(1-|w|^{2}\right)^{\beta} \frac{1-|\varphi(w)|^{2}}{(1-\overline{\varphi(w)} \psi(w))^{\alpha+1}} \psi^{\prime}(w)\right| \\
& \geq \frac{1}{2^{\alpha+1}}\left(\left|\mathscr{D}^{\alpha, \beta} \varphi(w)\right|-\left|\mathscr{D}^{\alpha, \beta} \psi(w) \frac{\left(1-|\varphi(w)|^{2}\right)\left(1-|\psi(w)|^{2}\right)^{\alpha}}{(1-\overline{\varphi(w)} \psi(w))^{\alpha+1}}\right|\right)
\end{aligned}
$$

and

$$
\begin{aligned}
\infty & >\left\|\left(C_{\varphi}-C_{\psi}\right) g_{\varphi(w)}\right\|_{\beta}=\sup _{z \in \mathbb{D}}\left(1-|z|^{2}\right)^{\beta}\left|\left(\left(C_{\varphi}-C_{\psi}\right) g_{\varphi(w)}\right)^{\prime}(z)\right| \\
& \geq\left(1-|w|^{2}\right)^{\beta}\left|\left(\left(C_{\varphi}-C_{\psi}\right) g_{\varphi(w)}\right)^{\prime}(w)\right| \\
& =\frac{1}{2^{\alpha+1}}\left(1-|w|^{2}\right)^{\beta} \frac{1-|\varphi(w)|^{2}}{|1-\overline{\varphi(w)} \psi(w)|^{\alpha+1}}\left|\frac{\varphi(w)-\psi(w)}{1-\overline{\varphi(w)} \psi(w)}\right|\left|\psi^{\prime}(w)\right| \\
& =\frac{1}{2^{\alpha+1}}\left|\mathscr{D}^{\alpha, \beta} \psi(w) \frac{\left(1-|\varphi(w)|^{2}\right)\left(1-|\psi(w)|^{2}\right)^{\alpha}}{(1-\overline{\varphi(w)} \psi(w))^{\alpha+1}}\right| \rho(\varphi(w), \psi(w)) .
\end{aligned}
$$


Multiplying $\rho(\varphi(w), \psi(w))$ on (2.2), combining with (2.3), we get

$$
\sup _{w \in \mathbb{D} \backslash \mathbb{D}_{1}}\left|\mathscr{D}^{\alpha, \beta} \varphi(w)\right| \rho(\varphi(w), \psi(w))<\infty
$$

where $\mathbb{D}_{1}=\{w \in \mathbb{D}: \varphi(w)=0\}$.

Since $\left|\mathscr{D}^{\alpha, \beta} \varphi(w)\right| \rho(\varphi(w), \psi(w))$ is continuous on $\mathbb{D}$ and $\mathbb{D}_{1}$ is dense in $\mathbb{D}$, we obtain

$$
\sup _{w \in \mathbb{D}}\left|\mathscr{D}^{\alpha, \beta} \varphi(w)\right| \rho(\varphi(w), \psi(w))<\infty
$$

For $w \in \mathbb{D}$ with $\psi(w) \neq 0$, using (2.1), we have

$$
\begin{aligned}
\infty & >|| C_{\varphi}-C_{\psi}\|\geq\|\left(C_{\varphi}-C_{\psi}\right) f_{\psi(w)}\left|\|_{\beta}=\sup _{z \in \mathbb{D}}\left(1-|z|^{2}\right)^{\beta}\right|\left(\left(C_{\varphi}-C_{\psi}\right) f_{\psi(w)}\right)^{\prime}(z) \mid \\
& \geq\left(1-|w|^{2}\right)^{\beta}\left|\left(\left(C_{\varphi}-C_{\psi}\right) f_{\psi(w)}\right)^{\prime}(w)\right| \\
& =\frac{1}{2^{\alpha+1}}\left|\left(1-|w|^{2}\right)^{\beta} \frac{1-|\psi(w)|^{2}}{\left(1-|\psi(w)|^{2}\right)^{\alpha+1}} \psi^{\prime}(w)-\left(1-|w|^{2}\right)^{\beta} \frac{1-|\psi(w)|^{2}}{(1-\overline{\psi(w)} \varphi(w))^{\alpha+1}} \varphi^{\prime}(w)\right| \\
\geq & \frac{1}{2^{\alpha+1}}\left|\mathscr{D}^{\alpha, \beta} \varphi(w)-\mathscr{D}^{\alpha, \beta} \psi(w)\right|-\left|\mathscr{D}^{\alpha, \beta} \varphi(w)\right|\left|\frac{1}{2^{\alpha+1}}-\frac{\left(1-|\psi(w)|^{2}\right)\left(1-|\varphi(w)|^{2}\right)^{\alpha}}{2^{\alpha+1}(1-\overline{\psi(w)} \varphi(w))^{\alpha+1}}\right| \\
& =\frac{1}{2^{\alpha+1}}\left|\mathscr{D}^{\alpha, \beta} \varphi(w)-\mathscr{D}^{\alpha, \beta} \psi(w)\right| \\
& -\left|\mathscr{D}^{\alpha, \beta} \varphi(w)\right|\left|\left(1-|\varphi(w)|^{2}\right)^{\alpha} f_{\psi(w)}^{\prime}(\varphi(w))-\left(1-|\psi(w)|^{2}\right)^{\alpha} f_{\psi(w)}^{\prime}(\psi(w))\right| \\
\geq & \frac{1}{2^{\alpha+1}}\left|\mathscr{D}^{\alpha, \beta} \varphi(w)-\mathscr{D}^{\alpha, \beta} \psi(w)\right|-c\left|\mathscr{D}^{\alpha, \beta} \varphi(w)\right| \rho(\varphi(w), \psi(w)) .
\end{aligned}
$$

Combining this with (2.5), we have

$$
\sup _{w \in \mathbb{D} \backslash \mathbb{D}_{2}}\left|\mathscr{D}^{\alpha, \beta} \varphi(w)-\mathscr{D}^{\alpha, \beta} \psi(w)\right|<\infty,
$$

where $\mathbb{D}_{2}=\{w \in \mathbb{D}: \psi(w)=0\}$, so we obtain

$$
\sup _{w \in \mathbb{D}}\left|\mathscr{D}^{\alpha, \beta} \varphi(w)-\mathscr{D}^{\alpha, \beta} \psi(w)\right|<\infty .
$$


(3) implies (4). We have

$$
\begin{aligned}
& \sup _{z \in \mathbb{D}}\left|\mathscr{D}^{\alpha, \beta} \psi(z)\right| \rho(\varphi(z), \psi(z)) \\
& \leq \sup _{z \in \mathbb{D}}\left|\mathscr{D}^{\alpha, \beta} \varphi(z)-\mathscr{D}^{\alpha, \beta} \psi(z)\right| \rho(\varphi(z), \psi(z))+\sup _{z \in \mathbb{D}}\left|\mathscr{D}^{\alpha, \beta} \varphi(z)\right| \rho(\varphi(z), \psi(z)) \\
& \leq \sup _{z \in \mathbb{D}}\left|\mathscr{D}^{\alpha, \beta} \varphi(z)-\mathscr{D}^{\alpha, \beta} \psi(z)\right|+\sup _{z \in \mathbb{D}}\left|\mathscr{D}^{\alpha, \beta} \varphi(z)\right| \rho(\varphi(z), \psi(z)) \\
& <\infty .
\end{aligned}
$$

(4) implies (1). For $f \in \mathscr{B}^{\alpha}$ with $\|f\|_{\alpha} \leq 1$, we have

$$
\begin{aligned}
& \|\left(C_{\varphi}-C_{\psi}\right) f \|_{\beta}=\sup _{z \in \mathbb{D}}\left|\left(1-|z|^{2}\right)^{\beta} f^{\prime}(\varphi(z)) \varphi^{\prime}(z)-\left(1-|z|^{2}\right)^{\beta} f^{\prime}(\psi(z)) \psi^{\prime}(z)\right| \\
&= \sup _{z \in \mathbb{D}}\left|\mathscr{D}^{\alpha, \beta} \varphi(z)\left(1-|\varphi(z)|^{2}\right)^{\alpha} f^{\prime}(\varphi(z))-\mathscr{D}^{\alpha, \beta} \psi(z)\left(1-|\psi(z)|^{2}\right)^{\alpha} f^{\prime}(\psi(z))\right| \\
& \leq \sup _{z \in \mathbb{D}}\left|\mathscr{D}^{\alpha, \beta} \varphi(z)-\mathscr{D}^{\alpha, \beta} \psi(z)\right|\left(1-|\varphi(z)|^{2}\right)^{\alpha}\left|f^{\prime}(\varphi(z))\right| \\
&+\sup _{z \in \mathbb{D}}\left|\mathscr{D}^{\alpha, \beta} \psi(z)\right|\left|\left(1-|\varphi(z)|^{2}\right)^{\alpha} f^{\prime}(\varphi(z))-\left(1-|\psi(z)|^{2}\right)^{\alpha} f^{\prime}(\psi(z))\right| \\
& \leq \sup _{z \in \mathbb{D}}\left|\mathscr{D}^{\alpha, \beta} \varphi(z)-\mathscr{D}^{\alpha, \beta} \psi(z)\right|+c \sup _{z \in \mathbb{D}}\left|\mathscr{D}^{\alpha, \beta} \psi(z)\right| \rho(\varphi(z), \psi(z)) \\
&<\infty,
\end{aligned}
$$

where we use Lemma 2.1 in the last second inequality. The proof of Theorem 2.1 is finished.

Remark 2.1. Note that when $\alpha=\beta=1,\left|\frac{1-|z|^{2}}{1-|\varphi(z)|^{2}} \varphi^{\prime}(z)\right| \leq 1$ for $\varphi \in S(\mathbb{D})$ by the Schwarz-Pick lemma. Then Theorem 2.1 implies that $C_{\varphi}-C_{\psi}$ is bounded on Bloch space $\mathscr{B}$.

\section{Compactness of $C_{\varphi}-C_{\psi}$}

In this section, we consider the compactness of $C_{\varphi}-C_{\psi}$ from $\mathscr{B}^{\alpha}\left(\mathscr{B}_{0}^{\alpha}\right)$ to $\mathscr{B}^{\beta}$ for $0<\alpha$, $\beta<\infty$. We first state some known theorems on composition operators on $\mathscr{B}^{\alpha}$ which shall be used latter. 
Lemma 3.1. ([2, Theorem 3.1]) If $0<\alpha, \beta<\infty$ and $\varphi \in S(\mathbb{D})$, then $C_{\varphi}: \mathscr{B}^{\alpha} \rightarrow \mathscr{B}^{\beta}$ is bounded if and only if

$$
\sup _{z \in \mathbb{D}}\left|\mathscr{D}^{\alpha, \beta} \varphi(z)\right|<\infty .
$$

Lemma 3.2. ([2, Theorem 4.2]) If $0<\alpha, \beta<\infty$ and $\varphi \in S(\mathbb{D})$, then $C_{\varphi}: \mathscr{B}^{\alpha} \rightarrow \mathscr{B}^{\beta}$ is compact if and only if

$$
\lim _{|\varphi(z)| \rightarrow 1}\left|\mathscr{D}^{\alpha, \beta} \varphi(z)\right|=0
$$

and

$$
\sup _{z \in \mathbb{D}}\left|\mathscr{D}^{\alpha, \beta} \varphi(z)\right|<\infty .
$$

By a similar proof of Proposition 3.11 in [3] we have the following result.

Lemma 3.3. If $0<\alpha, \beta<\infty$ and $\varphi, \psi \in S(\mathbb{D})$, then $C_{\varphi}-C_{\psi}: \mathscr{B}^{\alpha} \rightarrow \mathscr{B}^{\beta}$ is compact if and only if $\left\|\left(C_{\varphi}-C_{\psi}\right) f_{n}\right\|_{\beta} \rightarrow 0$ for any bounded sequence $\left\{f_{n}\right\}$ in $\mathscr{B}^{\alpha}$ that converges uniformly to 0 on every compact subset of $\mathbb{D}$.

For $\varphi \in S(\mathbb{D})$, let $\Gamma(\varphi)$ be the family of sequences in $\mathbb{D}$ such that $\left|\varphi\left(z_{n}\right)\right| \rightarrow 1$. We denote by $\mathbb{D}_{\alpha, \beta}(\varphi)$ the set of $\left\{z_{n}\right\}$ in $\Gamma(\varphi)$ such that $\mathscr{D}^{\alpha, \beta} \varphi\left(z_{n}\right) \rightarrow c \in \mathbb{C}$ with $c \neq 0$. For $\psi \in S(\mathbb{D})$, similarly we may define $\Gamma(\psi)$ and $\mathbb{D}_{\alpha, \beta}(\psi)$.

Theorem 3.1. Let $0<\alpha, \beta<\infty$ and $\varphi, \psi \in S(\mathbb{D})$. Suppose that $C_{\varphi}, C_{\psi}: \mathscr{B}^{\alpha} \rightarrow \mathscr{B}^{\beta}$ are bounded and neither of them are compact. Then the following statements are equivalent:

(1) $C_{\varphi}-C_{\psi}: \mathscr{B}^{\alpha} \rightarrow \mathscr{B}^{\beta}$ is compact;

(2) $C_{\varphi}-C_{\psi}: \mathscr{B}_{0}^{\alpha} \rightarrow \mathscr{B}^{\beta}$ is compact;

(3) $\mathbb{D}_{\alpha, \beta}(\varphi)=\mathbb{D}_{\alpha, \beta}(\psi) \neq \varnothing$ and for $\left\{z_{n}\right\} \in \Gamma(\varphi) \cap \Gamma(\psi)$,

$$
\begin{gathered}
\lim _{n \rightarrow \infty}\left|\mathscr{D}^{\alpha, \beta} \varphi\left(z_{n}\right)-\mathscr{D}^{\alpha, \beta} \psi\left(z_{n}\right)\right|=\lim _{n \rightarrow \infty}\left|\mathscr{D}^{\alpha, \beta} \varphi\left(z_{n}\right)\right| \rho\left(\varphi\left(z_{n}\right), \psi\left(z_{n}\right)\right) \\
=\lim _{n \rightarrow \infty}\left|\mathscr{D}^{\alpha, \beta} \psi\left(z_{n}\right)\right| \rho\left(\varphi\left(z_{n}\right), \psi\left(z_{n}\right)\right)=0 .
\end{gathered}
$$

Proof. It's obvious that (1) imples (2).

(2) implies (3). Since $C_{\varphi}: \mathscr{B}^{\alpha} \rightarrow \mathscr{B}^{\beta}$ is bounded and noncompact, by Lemmas 3.1 and 3.2 we have $\mathbb{D}_{\alpha, \beta}(\varphi) \neq \varnothing$. To show $\mathbb{D}_{\alpha, \beta}(\varphi) \subset \mathbb{D}_{\alpha, \beta}(\psi)$, let $\left\{z_{n}\right\} \in \mathbb{D}_{\alpha, \beta}(\varphi)$. Since $\left|\varphi\left(z_{n}\right)\right| \rightarrow 1$, we may assume that $\varphi\left(z_{n}\right) \neq 0$ for every $n$. For $\lambda \in \mathbb{D}$ with $\lambda \neq 0$, define $f_{\lambda}, g_{\lambda}$ as in the proof of Theorem 2.1. We know that $f_{\lambda}, g_{\lambda} \in \mathscr{B}_{0}^{\alpha}$ and the sequences $\left\{f_{\varphi\left(z_{n}\right)}\right\}$ and $\left\{f_{\psi\left(z_{n}\right)}\right\}$ are bounded in $\mathscr{B}^{\alpha}$ and converge to 0 uniformly on every compact subset of $\mathbb{D}$ as $n \rightarrow \infty$. From Lemma 3.3,

$$
\begin{aligned}
0 & \leftarrow\left\|\left(C_{\varphi}-C_{\psi}\right) f_{\varphi\left(z_{n}\right)}\right\|_{\beta} \geq\left(1-\left|z_{n}\right|^{2}\right)^{\beta}\left|\left(\left(C_{\varphi}-C_{\psi}\right) f_{\varphi\left(z_{n}\right)}\right)^{\prime}\left(z_{n}\right)\right| \\
& \geq \frac{1}{2^{\alpha+1}}\left(\left|\mathscr{D}^{\alpha, \beta} \varphi\left(z_{n}\right)\right|-\left|\mathscr{D}^{\alpha, \beta} \psi\left(z_{n}\right) \frac{\left(1-\left|\varphi\left(z_{n}\right)\right|^{2}\right)\left(1-\left|\psi\left(z_{n}\right)\right|^{2}\right)^{\alpha}}{\left(1-\overline{\varphi\left(z_{n}\right)} \psi\left(z_{n}\right)\right)^{\alpha+1}}\right|\right),
\end{aligned}
$$




$$
\begin{aligned}
0 & \leftarrow\left\|\left(C_{\varphi}-C_{\psi}\right) g_{\varphi\left(z_{n}\right)}\right\|_{\beta} \geq\left(1-\left|z_{n}\right|^{2}\right)^{\beta}\left|\left(\left(C_{\varphi}-C_{\psi}\right) g_{\varphi\left(z_{n}\right)}\right)^{\prime}\left(z_{n}\right)\right| \\
& =\frac{1}{2^{\alpha+1}}\left|\mathscr{D}^{\alpha, \beta} \psi\left(z_{n}\right) \frac{\left(1-\left|\varphi\left(z_{n}\right)\right|^{2}\right)\left(1-\left|\psi\left(z_{n}\right)\right|^{2}\right)^{\alpha}}{\left(1-\overline{\varphi\left(z_{n}\right)} \psi\left(z_{n}\right)\right)^{\alpha+1}}\right| \rho\left(\varphi\left(z_{n}\right), \psi\left(z_{n}\right)\right)
\end{aligned}
$$

Multiplying $\rho\left(\varphi\left(z_{n}\right), \psi\left(z_{n}\right)\right)$ on (3.1), noting that (3.2), we get

$$
\lim _{n \rightarrow \infty}\left|\mathscr{D}^{\alpha, \beta} \varphi\left(z_{n}\right)\right| \rho\left(\varphi\left(z_{n}\right), \psi\left(z_{n}\right)\right)=0 .
$$

Since $\left\{z_{n}\right\} \in \mathbb{D}_{\alpha, \beta}(\varphi), \rho\left(\varphi\left(z_{n}\right), \psi\left(z_{n}\right)\right) \rightarrow 0$. Since $\left|\varphi\left(z_{n}\right)\right| \rightarrow 1$, we have $\left\{z_{n}\right\} \in \Gamma(\psi)$ and may assume that $\psi\left(z_{n}\right) \neq 0$ for every $n$.

Similar to (2.6), we have

$$
\begin{aligned}
0 & \leftarrow\left\|\left(C_{\varphi}-C_{\psi}\right) f_{\psi\left(z_{n}\right)}\right\|_{\beta} \\
& \geq \frac{1}{2^{\alpha+1}}\left|\mathscr{D}^{\alpha, \beta} \varphi\left(z_{n}\right)-\mathscr{D}^{\alpha, \beta} \psi\left(z_{n}\right)\right|-c\left|\mathscr{D}^{\alpha, \beta} \varphi\left(z_{n}\right)\right| \rho\left(\varphi\left(z_{n}\right), \psi\left(z_{n}\right)\right) .
\end{aligned}
$$

Hence by (3.3), we get

$$
\lim _{n \rightarrow \infty}\left|\mathscr{D}^{\alpha, \beta} \varphi\left(z_{n}\right)-\mathscr{D}^{\alpha, \beta} \psi\left(z_{n}\right)\right|=0
$$

and

$$
\lim _{n \rightarrow \infty}\left|\mathscr{D}^{\alpha, \beta} \psi\left(z_{n}\right)\right| \rho\left(\varphi\left(z_{n}\right), \psi\left(z_{n}\right)\right)=\lim _{n \rightarrow \infty}\left|\mathscr{D}^{\alpha, \beta} \varphi\left(z_{n}\right)\right| \rho\left(\varphi\left(z_{n}\right), \psi\left(z_{n}\right)\right)=0
$$

Since $\mathscr{D}^{\alpha, \beta} \varphi\left(z_{n}\right) \rightarrow c \neq 0$, we have $\mathscr{D}^{\alpha, \beta} \psi\left(z_{n}\right) \rightarrow c$. Then $\left\{z_{n}\right\} \in \mathbb{D}_{\alpha, \beta}(\psi)$, so $\mathbb{D}_{\alpha, \beta}(\varphi) \subset$ $\mathbb{D}_{\alpha, \beta}(\psi)$. Similarly, $\mathbb{D}_{\alpha, \beta}(\psi) \subset \mathbb{D}_{\alpha, \beta}(\varphi)$. Hence $\mathbb{D}_{\alpha, \beta}(\varphi)=\mathbb{D}_{\alpha, \beta}(\psi)$.

Let $\left\{z_{n}\right\} \in \Gamma(\varphi) \cap \Gamma(\psi)$ such that $\mathscr{D}^{\alpha, \beta} \varphi\left(z_{n}\right) \rightarrow 0$. We have

$$
\lim _{n \rightarrow \infty}\left|\mathscr{D}^{\alpha, \beta} \varphi\left(z_{n}\right)\right| \rho\left(\varphi\left(z_{n}\right), \psi\left(z_{n}\right)\right)=0
$$

and by (3.4),we obtain

$$
\lim _{n \rightarrow \infty}\left|\mathscr{D}^{\alpha, \beta} \varphi\left(z_{n}\right)-\mathscr{D}^{\alpha, \beta} \psi\left(z_{n}\right)\right|=0 .
$$

Thus

$$
\lim _{n \rightarrow \infty}\left|\mathscr{D}^{\alpha, \beta} \psi\left(z_{n}\right)\right|=\lim _{n \rightarrow \infty}\left|\mathscr{D}^{\alpha, \beta} \varphi\left(z_{n}\right)\right|=0
$$

and

$$
\lim _{n \rightarrow \infty}\left|\mathscr{D}^{\alpha, \beta} \psi\left(z_{n}\right)\right| \rho\left(\varphi\left(z_{n}\right), \psi\left(z_{n}\right)\right)=0 .
$$

Combing this with(3.5)and(3.6), we get (3).

(3) implies (1). This can be proved by a similar way to the proof of Theorem 3.2 in [4]. From (3.3) and the fact that $\left|\mathscr{D}^{\alpha, \beta} \varphi\left(z_{n}\right)\right| \nrightarrow 0$. we obtain. 


\section{Acknowledgments}

The author would like to thank her tutor Professor Zengjian Lou for his long-term careful guidance and the referees for their helpful comments on this paper. The work is supported by the Young and Middle-aged Education and Scientific Research Project of Fujian Province (Grant No. 2015 JA15734).

\section{References}

[1] P.J. Nieminen. Compact differences of composition operators on Bloch and Lipschitz spaces. Comput. Methods Funct. Theory, to appear.

[2] Z.J. Lou. Composition operators on Bloch type spaces. Analysis, 2003, 23: 81-95.

[3] C.C. Cowen, B.D. MaCcluer. Composition operators on spaces of analytic functions. CRC Press, Boca Raton 1995.

[4] T. Hosokawa, S. Ohno. Differences of composition operators on the Bloch spaces. J. Operator Theory, 2007, 57: 229-242.

[5] K. Madigan, A. Matheson. Compact composition operators on the Bloch space. Trans. Amer. Math. Soc., 1995, 347: 2679-2687.

[6] S. Ohno, K. Stroethoff, R.H. Zhao. Weighted composition operators between Bloch-type spaces. Rocky Mountain J. Math., 2003, 33: 191-215.

[7] K. Zhu. Bloch type spaces of analytic functions. Rocky Mountain J. Math., 1993, 23: 11431177.

[8] Q. Huang. The boundness of differences of composition operators on little Bloch type space. Journal of Nanchang College of Education, 2013, 12: 69-71. 